\title{
N/SA News
}

Natıonal Aeronautics and

Space Admınıstratıon

Washıngton DC 20546

AC 202 755-8370

$p 81-10033$

Charles Redmond

For Release

Headquarters, Washington, D.C.

(Phone: 202/755-3680)

Peter Waller

Ames Research Center, Mountain View, Calif. (Phone: 415/965-5091)

RELEASE NO: $81-33$

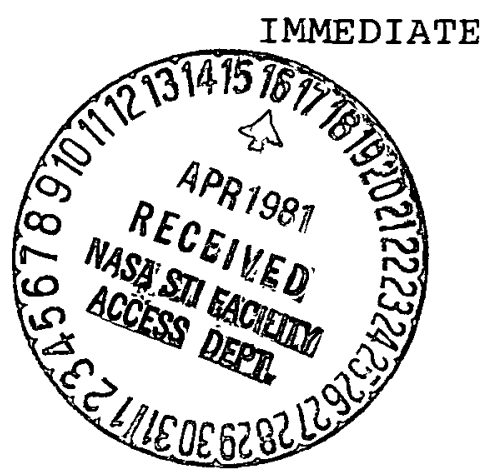

VENUS CLOUD STUDIES SHOW CHANGES IN LONG-TERM WIND PATTERNS

Significant discoveries concerning the pattern of atmospheric changes on venus have been made based on two years' worth of Pioneer orbiter observations. The discoveries indicate a long term period of change for both the planet's wind patterns and for the existence of a haze layer above the cloud tops.

The Pioneer orbiter has taken about 1,000 pictures of venus clouds and extensive measurements of the particles composing those clouds.

The most noteworthy of the discoveries is that Venus' planet-wide wind patterns change dramatically over a period of several years. Two patterns have been discernea -- a mid-latitude jet stream pattern and a cloud and wind pattern which acts like a solid body.

March 9, 1981 
A second discovery shows that the high-altitude haze layer which completely envelopes Venus' clouds appears and disappears over several-year periods. This haze is a "smog layer" extending above the main cloud region by about 30 kilometers (18 miles). This altitude on the Earth would be well into our stratosphere.

The Pioneer Venus orbiter is expected to return pictures and other data until 1985. The orbiter reached Venus in December 1978, and the four Pioneer probe craft entered the atmosphere at the same time. Cloud pictures and polarimetry data are provided by the cloud photopolarimeter. Dr. Larry Travis, NASA Goddard Institute for space studies, New York City, is principal investigator.

The two new discoveries of a multi-year change in the pattern of global winds, and similar changes in the planet-wrapping haze layer could help explain the major remaining mystery of venus' atmosphere: Why, on a planet which has almost no axial rotation, do the upper level winds circle the planet at tremendous speeds of 360 kilometers per hour (225 miles per hour). These winds cover the planet completely, blowing at virtually every latitude from equator to pole. Their speeds can be determined from the speeds at which the clouds, carried by the winds, travel around the planet.

Wind speed measurements from top to bottom of the atmosphere by the four Pioneer probe craft show that these high-speed, cloudlevel winds are coupled to lower altitude winds, which also have very high speeds. 
The $360 \mathrm{~km} / \mathrm{hr}(225 \mathrm{mph})$ cloud level winds blow around the planet at an altitude of $65 \mathrm{~km}(40 \mathrm{mi})$. Wind speeds then range down to $192 \mathrm{~km} / \mathrm{hr}$ (120 mph) at $50 \mathrm{~km}$ (30 mi.) altitude and to a still-very-high $80 \mathrm{~km} / \mathrm{hr}(50 \mathrm{mph})$ at $20 \mathrm{~km}(12 \mathrm{mi.})$ altitude.

The mass of the moving atmosphere constituting these highspeed winds is several times that of the entire atmosphere. It represents about a quarter of Venus' atmosphere which is about 100 times denser than Earth's.

Despite the scale of these high-speed, upper level winds, well over half of Venus' tremendously dense atmosphere, near the planet's surface, is almost stagnant. From the surface up to $10 \mathrm{~km}$ ( $6 \mathrm{mi}$.$) altitude, wind speeds are only about 3$ to $18 \mathrm{~km} / \mathrm{hr}$ (2 to $11 \mathrm{mph}$ ).

In a general way, the high-speed winds can now be explained as due to the transfer of momentum from Venus' slow-moving, massive lower atmosphere to higher altitudes where the atmosphere is less massive, so that the same momentum results in a much higher velocity.

These new discoveries of long-term changes in global wind patterns and an enormous haze envelope which appears and disappears could help scientists further define the "driver" for the planet's high-speed winds. Any future general atmosphere circulation model for venus will have to produce these long-term changes in wind and cloud patterns. 
Details of these and other major findings from the two-year analysis of the venus cloud and polarimetry data include:

- It is now clear that the high-speed movements of Venus' clolids around the planet are not caused by wave motions in the atmosphere, as was thought by a number of scientists, but are real winds, though there are some wave motions as well. These planet-circling winds, which carry along the clouds, are the same ones which were measured by the four pioneer venus probe craft as they descended to Venus' surface in December 1978. These winds blow in an east to west direction, circling the planet once every four days at speeds near the equator of $360 \mathrm{~km} / \mathrm{hr}(225 \mathrm{mph})$, and near the poles (at around 70 degrees latitude) of $160 \mathrm{~km} / \mathrm{hr}$ (100 mph). The pioneer cloud pictures show the region of Venus' main cloud deck at altitudes between 60 and $65 \mathrm{~km}$ ( 37 and $40 \mathrm{mi}$.) above the planet's surface.

- The global pattern of these planet-circling, cloud-level winds appears to change periodically. For the past two years of Pioneer observations, Venus' clouds and cloud-level winds have been evidencing "solid body" rotation. That is, they move around Venus as though they were made up of one solid, planet-encasing body. This pattern of motion, of course, means wind speeds are much higher at the equator than at the poles.

- However, in 1974 when the Mariner spacecraft flew past Venus, the clouds did not circle the planet as a solid body, and there were mid-latitude jet streams at around 45 degrees lati tude. 
These higher-speed winds had velocities of around $400 \mathrm{~km} / \mathrm{hr}$ (250 mph), while wind velocities at the equator were some $40 \mathrm{~km} / \mathrm{hr}$ (25 mph) lower, at $360 \mathrm{~km} / \mathrm{hr}(225 \mathrm{mph})$.

This seems to indicate that there is an irregular cycle of change in the pattern of these cloud-level winds -- perhaps several years in length. The duration and rate of change of this cycle of changing wind patterns would be of fundamental interest in understanding the high-speed flow of Venus' upper level winds around the planet, as well as the behavior of the general atmosphere circulation.

- Measurements of Venus' cloud level winds show that, in addition to circling the planet, they also blow toward the poles at speeds of around $25 \mathrm{~km} / \mathrm{hr}$ (15 mph). These equator-to-pole winds (also seen by the four pioneer probes at lower altitudes) carry heat from the sun, absorbed near the venus equator, to the poles.

The speeds of these equator-to-pole winds agree with the wind measurements by the four Pioneer probes. This indicates that the cloud-level winds are the upper limb of an equator-topole Hadley cell circulation loop, which carries Venus' equatorial heat poleward.

- The so-called global "Y" pattern of Venus clouds with the tail of the "Y" extending eastward around the planet, and the arms westward, appears at times, but is not typical. The "Y" was first seen in ground observations. 
Sometimes the "Y", which occasionally extends two-thirds of the way around the planet, disappears completely. At other times, it is so changed that it forms a " $C$ " or other shape. In general, the planet shows a whole range of global cloud patterns in addition to the "Y".

- In addition to its well-known veil of clouds, two years of Pioneer polarimetry measurements show that Venus is currently enveloped in an 18-mile-thick blanket of high-altitude haze. The haze is present everywhere, but has about three times more particles ver unit volume at the poles than at the equator. At the poles, the haze is so thick that it obscures the base clouds beneath it. This haze of tiny sulfuric acid droplets is the "sealer" of Venus' greenhouse effect, holding additional heat beyond that which would be trapped by the clouds and atmosphere alone. The planet's 482 Celsius ( 900 Fahrenheit) surface temperature would be somewhat lower without the haze. Furthermore, inclusion of haze effects makes the Venus heat radiation models developed by scientists match the cloud top atmosphere structure observed by the Pioneer Venus instruments.

Venus' main clouds consist of sulfuric acid particles two microns in diameter, while in the haze layer, the particles are smaller, only a quarter of this size.

Over the next five years of the Pioneer Venus orbiter's extended mission (through 1985), Dr. Travis will be watching the clouds for changes. 
He will be looking for changes in the global circulation pattern, such as the one which occurred in the five years separating the Pioneer and Mariner missions (1974-1979). Determining such factors as the rapidity of the change and the frequency of its occurrence should lead to further understanding of the Venusian high-speed winds.

The Pioneer Project is managed by NASA's Ames Research Center, Mountain View, Calif. The spacecraft were built by Hughes Aircraft Co.

$$
\text { -end- }
$$

(Index: 23, 28, 33) 